\title{
Insights into Interprotein Electron Transfer of Human Cytochrome c Variants Arranged in Multilayer Architectures by Means of an Artificial Silica Nanoparticle Matrix
}

\author{
Sven Christian Feifel, ${ }^{* \dagger}{ }^{\dagger}$ Kai Ralf Stieger, ${ }^{\dagger}$ Andreas Kapp, ${ }^{\dagger}$ Dennis Weber, ${ }^{\dagger}$ Marco Allegrozzi, ${ }^{\ddagger}$ \\ Mario Piccioli, ${ }^{\ddagger}$ Paola Turano, ${ }^{\ddagger}$ and Fred Lisdat ${ }^{*}{ }^{\dagger}$ \\ ${ }^{\dagger}$ Institute of Applied Life Sciences, Biosystems Technology, Technical University of Applied Sciences Wildau, Hochschulring 1, 15745 \\ Wildau, Germany \\ ${ }^{\ddagger}$ Department of Chemistry and Magnetic Resonance Center, University of Florence, Via Luigi Sacconi 2, 50019 Sesto Fiorentino, \\ Florence, Italy
}

\section{Supporting Information}

\begin{abstract}
The redox behavior of proteins plays a crucial part in the design of bioelectronic systems. We have demonstrated several functional systems exploiting the electron exchange properties of the redox protein cytochrome $c$ (cyt $c$ ) in combination with enzymes and photoactive proteins. The operation is based on an effective reaction at modified electrodes but also to a large extent on the capability of self-exchange between cyt $c$ molecules in a surface-fixed state. In this context, different variants of human cyt $c$ have been examined here with respect to an altered heterogeneous electron transfer (ET) rate in a monolayer on electrodes as well as an enhanced self-exchange rate while being incorporated in multilayer architectures. For this purpose, mutants of the wild-type (WT) protein have been prepared to change the chemical nature of the surface contact area near the heme edge. The structural integrity of the variants has been verified by NMR and UV-vis measurements. It is shown that the single-point mutations can significantly influence the heterogeneous ET rate at thiol-modified gold electrodes and that electroactive protein/silica nanoparticle multilayers can be constructed with all forms of human cyt $c$ prepared. The kinetic behavior of electron exchange for the mutant proteins in comparison with that of the WT has been found altered in some multilayer arrangements. Higher self-exchange rates have been found for K79A. The results demonstrate that the position of the introduced change in the charge situation of cyt $c$ has a profound influence on the exchange behavior. In addition, the behavior of the cyt $c$ variants in assembled multilayers is found to be rather similar to the situation of cyt $c$ self-exchange in solution verified by NMR.
\end{abstract}

\section{INTRODUCTION}

Immobilization of redox proteins on electrodes has received rising notice over the last two decades from both elementary and practical points of view. The specific characteristics of proteins and enzymes such as molecular recognition, redox properties, and catalytic features provide a profound basis for the development of bioelectronic devices such as biofuel cells and biosensors. ${ }^{1,2}$ Redox proteins have gained considerable attention with this respect; their use originates to a large extend from specific reactions with biomolecular interaction partners but also with small molecules involved in oxidative stress such as hydrogen peroxide, ${ }^{3,4}$ superoxide, $^{5-7}$ or nitric oxide. ${ }^{8}$ Examples are myoglobin, ${ }^{3}$ hemoglobin, ${ }^{4}$ azurin, ${ }^{7}$ cytochrome $c^{\prime}$ (prime) (cyt $\left.c^{\prime}\right),{ }^{8}$ cytochrome $c$ (cyt $\left.c\right) .{ }^{5,6}$

In this regard, mitochondrial cyt $c$ has been extensively used as a model redox protein due to the stability and availability of this well-characterized soluble heme protein. ${ }^{9}$ Several routes have been designed to constitute cyt $c$ monolayers on different electrodes with variable performance. ${ }^{10-13}$ For metal electro- des, often self-assembly monolayers (SAM) are used for modification. Cyt $c$ can be electrostatically assembled, for example, on carboxy-terminated alkanethiols and/or can also be covalently coupled. ${ }^{14}$ Furthermore, it has been demonstrated that the immobilized redox protein can be reduced by superoxide radicals, whereupon the protein is reoxidized by the electrode, resulting in a current that is proportional to the radical concentration. ${ }^{15-19}$ The performance of such a sensing system can be improved by increasing the protein concentration by means of protein multilayers. ${ }^{20}$

Multilayer architectures of the redox protein can be prepared through the layer-by-layer deposition of cyt $c$ and different kinds of second building blocks, such as polyelectrolytes, ${ }^{21}$ $\mathrm{DNA}^{22}$ gold, $^{23}$ and silica nanoparticles (SiNPs). ${ }^{24}$

Received: August 29, 2016

Accepted: November 4, 2016

Published: December 1, 2016 
Communication within such architectures, however, is a vital issue, as the electron transfer (ET) pathways in the assemblies significantly increase with the number of deposited layers. ${ }^{25}$ It has been shown that ET in the multilayer systems occurs through protein-protein electron self-exchange among the cyt $c$ molecules. ${ }^{21,26}$ Charge transfer proceeds thus by an electron hopping process among the heme centers confined in a protein shell (with many positive charges at neutral $\mathrm{pH}^{27}$ ). This mechanism may require some rotational flexibility of the cyt $c$ molecules embedded in the multilayer architecture.

A further advanced stage is shown by embedding additional biomolecules, such as enzymes, into cyt $c$ multilayers to create artificial biprotein signal chains. ${ }^{2,17,28-30}$ Cyt $c$ operates in these systems as a solid-state ET protein, facilitating ET between the enzyme and the electrode without any external mediator. It has been shown that the reaction at the enzyme becomes the ratedetermining step at low scan rates. However, it was also found that at higher scan rates the supply or withdrawal of electrons to or from the enzyme becomes important indicating limitations by the protein-protein exchange. ${ }^{17}$ One approach for examining alterations in the protein self-exchange reaction rate can be seen in protein engineering. NMR is a valuable method for analyzing self-exchange rates and thus has been often used for evaluating exchange reactions among redox proteins in solution. ${ }^{31-35}$

Mutational studies of ET proteins have been utilized to understand the reaction with other biomolecules or to locate protein interaction sites. Previously, it has been shown that mutations of cyt $c$ can affect the reaction with enzymes, for example, the reaction with cyt $c$ oxidase. ${ }^{36}$ Furthermore, superoxide sensors have been constructed on the basis of human cyt $c$ mutants, showing that the sensitivity can be increased due to the raised reaction rate with the oxygen radical by means of amino acid exchange. ${ }^{37}$

Here, we examine different variants of human cyt $c$ in which a positively charged lysine residue is replaced by a neutral alanine residue with respect to the protein-protein self-exchange reaction rate $\left(k_{\mathrm{ex}}\right)$ and the electrochemical behavior in an artificial SiNP-based multilayer matrix on modified gold electrodes. The investigation is dedicated to gain a more profound understanding of factors influencing the ET inside multilayer architectures, which are based on direct electron exchange between immobilized protein molecules. The primary focus is on chasing mutation points by which the self-exchange kinetics of cyt $c$ can be enhanced and thus providing a path to use these ET relays for improved electrochemical communication with biocatalysts in a multilayer system.

\section{RESULTS AND DISCUSSION}

In the present work, the self-exchange reaction and the electrochemical behavior of five human cyt $c$ mutants have been investigated in different SAM/cyt $c$ - $[\text { cyt } c \cdot S i N P s]_{4-6}$ multilayer architectures on gold electrodes by cyclic voltammetry (CV), surface plasmon resonance (SPR), and in solution by NMR. This approach allows, on the one hand, a monitoring of the electrochemical behavior (by $\mathrm{CV}$ ) and of the binding properties of the different mutants on a surface (via SPR); on the other hand, it correlates these findings with the structural properties of the mutants and the self-exchange in solution (measured by NMR).

Choice and Assessment of Human Cyt c Mutants. We choose human cyt $c$ to investigate the influence of amino acid residue modifications and to alter the protein-protein self- exchange reaction rate in a SiNP-based multilayer matrix. This analysis is based on reports showing that lowering net charges in cyt $c$ can lead to increased electron self-exchange rates ${ }^{38,40}$ and that the introduction of additional positively charged lysines close to the heme pocket leads to a decreased $k_{\text {ex }}{ }^{39}$

In general, ET reactions between biomolecules in solution require protein association followed by an ET. The value of the protein-protein association rate constant $\left(k_{\mathrm{on}}\right)$, which is determined by long-range association forces, can be augmented by favorable electrostatic interactions such as complementary charge distribution on the binding partners. ${ }^{41} \mathrm{~A}$ fast ET requires the formation of a highly specific productive complex, often in conjunction with a low-specificity encounter state. ${ }^{42}$ The encounter state has a transient and highly dynamic nature because it consists of an ensemble of interconverting relative orientations of the partner molecules. From the encounter state, the number of short-range interactions between the redox partners is increased to form the stereospecific state corresponding to the productive complex. ${ }^{43}$ For the formation of a productive complex, van der Waals interactions also play a relevant role, as observed, for example, for the interaction between cyt $c$ and cyt $c$ oxidase. ${ }^{44,45}$

In functional redox complexes involving cyt $c$, two critical interaction areas can be identified: (1) a central one, which involves hydrophobic residues and accounts for the most stable interaction, which is encased by (2) a ring of charged residues (mainly lysines) that give rise to transient interactions, switching on and off electrostatic salt bridges with different carboxylate residues on the facing partner; these interconverting salt bridges are the basis of the multiple conformations of the encounter complex. Single-point mutations at the interface can shift the equilibrium involving the formation of encounter and productive complexes. It is reasonable to assume that similar mechanisms could apply in self-exchange ET complexes, as in the present case-not only in solution, but also when cyt $c$ is in a quasi-immobilized state. Taking these aspects into account, five positively charged residues have been selected to be replaced with neutral alanine: Lys13Ala, Lys27Ala, Lys72Ala, Lys73Ala, Lys79Ala. In Figure 1, all of the chosen single-point

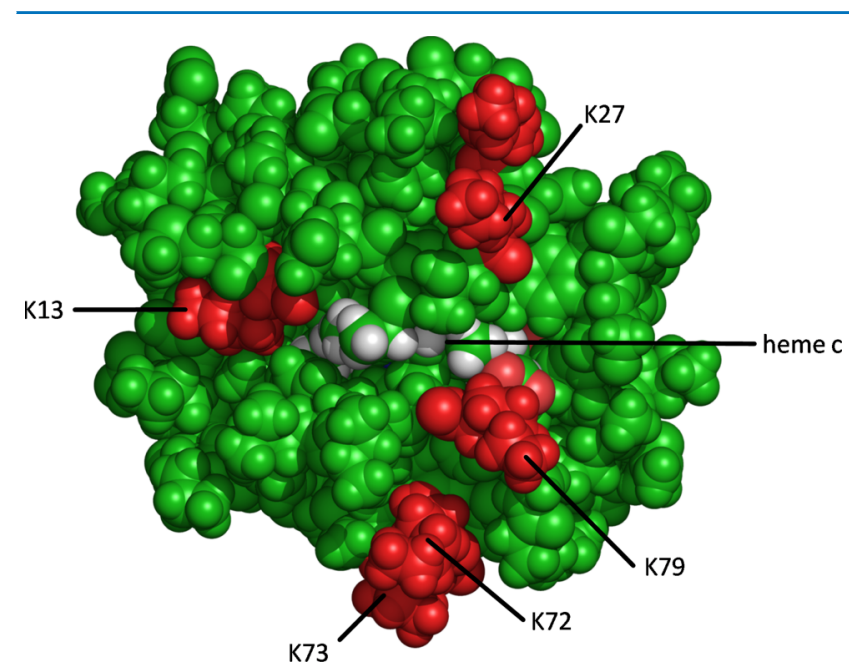

Figure 1. Structure of human cyt $c$ with mutation sites (for alanine) represented in red color. In the midpoint, the heme group is depicted in gray/green color. The structure has been generated with the PyMOL software (https://sourceforge.net/projects/pymol/), PDB file $1 \mathrm{~J} 3 \mathrm{~S}$. 
mutations at the cyt $c$ molecule are depicted. The chosen Lys mutation positions are surface exposed and solely located on one side of cyt $c$ with respect to the heme edge.

UV-Vis and NMR Spectroscopic Characterization of Human Cyt c. UV-vis absorption spectra of WT and variants of human cyt $c$ in its reduced form are shown in Figure S1. Virtually all of the investigated variants display their absorption maximums in the reduced state (Soret, $\alpha$, and $\beta$ band) at similar wavelengths compared to the WT protein. In the oxidized state, the absorption maxima of the $\gamma$ band of the mutants are also comparable to those of WT cyt $c$ (Table 1).

Table 1. Main Spectroscopic Features of the Human WT Cyt $c$ and Its Variant Forms

\begin{tabular}{cccc} 
& oxidized state maximum $(\mathrm{nm})$ & \multicolumn{2}{c}{$\begin{array}{c}\text { reduced state } \\
\text { maximum }(\mathrm{nm})\end{array}$} \\
human cyt $c$ form & $\gamma$ & $\gamma$ & $\alpha$ \\
WT & 410 & 415 & 550 \\
K13A & 410 & 414 & 550 \\
K27A & 410 & 414 & 550 \\
K72A & 410 & 414 & 550 \\
K73A & 410 & 414 & 550 \\
K79A & 409 & 414 & 550 \\
\hline
\end{tabular}

The coordination state of the ferric form of WT cyt $c$ and its variants has been inspected by paramagnetic monodimensional ${ }^{1} \mathrm{H}$ NMR over large spectral widths. The well-resolved hyperfine-shifted resonances of the cyt $c$ heme methyls, 8$\mathrm{CH}_{3}$ and $3-\mathrm{CH}_{3}$, and of the axial ligands for the iron atom (His18, $\mathrm{H} \delta 2$, and Met80 $\varepsilon-\mathrm{CH}_{3}$ ) are diagnostic for the electronic properties of the ferric heme center in cyt $c$ and are used as a fingerprint to assure the integrity of the heme environment for all cyt $c$ mutants (Figure 2). ${ }^{46,47}$ Resonance position and line widths are very similar in the WT and mutant proteins, pointing to a conserved low-spin ferric heme center with intact coordination. This is clearly apparent from the

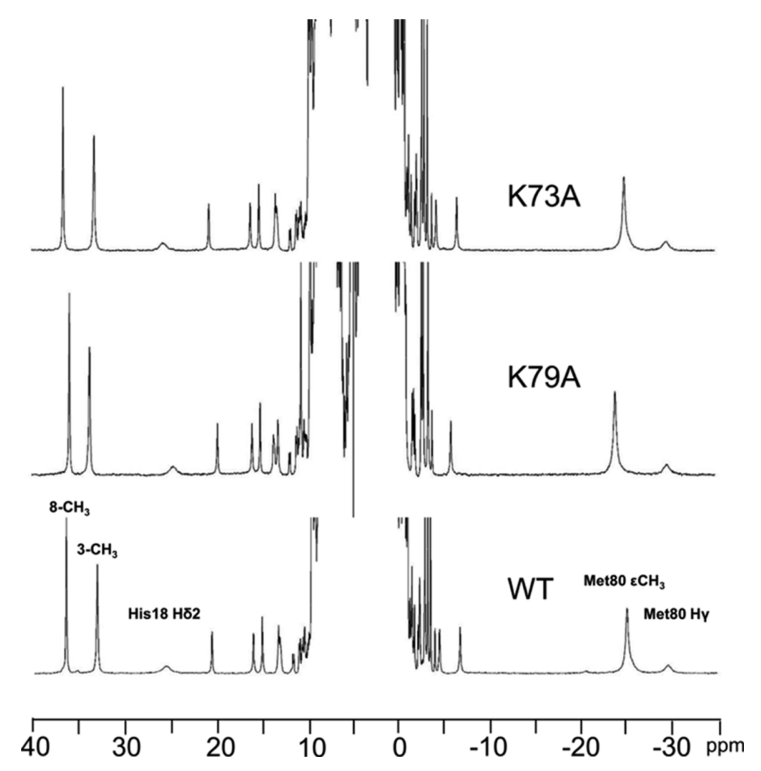

Figure 2. One-dimensional (1D) ${ }^{1} \mathrm{H}$ NMR spectra of the ferric forms of WT cyt $c$ and its variants (K73A, K79A), showing very similar signal patterns for the hyperfine-shifted resonances of the heme substituents and axial ligands. comparison reported in Figure 2 for the variants pinpointed by electrochemical analysis (vide infra). ${ }^{1} \mathrm{H}-{ }^{15} \mathrm{~N}$ heteronuclear single-quantum coherence (HSQC) experiments acquired for both oxidation states of some variants (data not shown) prove the intact fold. Indeed, the HSQC maps for the WT, K73A, and K79A variants in both ferric and ferrous forms are essentially superimposable, besides small chemical shift perturbations localized in the immediate surroundings of the mutation sites.

Determination of the Electrochemical Properties of Human Cyt c Variants in a Monolayer Arrangement. It has been examined whether the different cyt $c$ forms can be stably adsorbed on a thiol-modified Au electrode (mercaptoundecanoic acid/mercaptoundecanol (MUA/MU) is used here because of fast ET for cyt $c) .{ }^{20}$ To examine the electrochemical properties and for calculation of the heterogeneous ET rate constant $\left(k_{\mathrm{s}}\right)$ and the redox potentials, CV has been carried out at different scan rates. It has been found that the CVs of the mutant proteins are rather comparable to those of WT cyt $c$ (Figure S2). The collected data in terms of redox potentials and heterogeneous ET rate constants $\left(k_{\mathrm{s}}\right)$ are given in Table 2.

Table 2. Electrochemical Properties of Cyt $c$ Assembled on MU/MUA-Modified Au Electrodes, Assessed by CV Investigations (Formal Potential $\left(E_{\mathrm{f}}\right)^{a}$, Surface Coverage $(\Gamma)^{a}$, Half-Peak Width $\left(\mathrm{Ew}_{1 / 2}\right)^{a}$, and Heterogeneous ET Rate Constant $\left.\left(k_{\mathrm{s}}\right)\right)^{a}$

$\begin{array}{lcccc}\begin{array}{c}\text { human cyt } c \\ \text { forms }\end{array} & \begin{array}{c}E_{\mathrm{f}} \text { ads. } \\ (\mathrm{mV})\end{array} & \begin{array}{c}\Gamma \text { ads. } \\ \left(\mathrm{pmol} / \mathrm{cm}^{2}\right)\end{array} & \begin{array}{c}\mathrm{Ew}_{1 / 2} \text { ads. } \\ (\mathrm{mV})\end{array} & \begin{array}{c}k_{\mathrm{s}} \text { ads. } \\ \left(\mathrm{s}^{-1}\right)\end{array} \\ \text { WT } & -15 \pm 2 & 15 \pm 2 & 100 \pm 3 & 78 \pm 6 \\ \text { K13A } & -33 \pm 3 & 15 \pm 2 & 99 \pm 3 & 73 \pm 2 \\ \text { K27A } & -31 \pm 4 & 13 \pm 1 & 105 \pm 2 & 30 \pm 2 \\ \text { K72A } & -22 \pm 3 & 13 \pm 1 & 101 \pm 5 & 76 \pm 4 \\ \text { K73A } & -19 \pm 3 & 12 \pm 2 & 97 \pm 2 & 95 \pm 3 \\ \text { K79A } & 0 \pm 4 & 10 \pm 2 & 97 \pm 4 & 69 \pm 5\end{array}$

${ }^{a}$ All values are evaluated as the average value of at least three electrodes. Formal potentials $\left(E_{\mathrm{f}}\right)$ were evaluated by the use of an $\mathrm{Ag} /$ $\mathrm{AgCl}$ reference electrode with $1 \mathrm{M} \mathrm{KCl}$.

For the investigated mutants, only small variations in the redox potential have been observed in the adsorbed state. Representative CVs of the WT cyt $c$ and mutant K79A are shown in Figure 3. The formal redox potentials $\left(E_{\mathrm{f}}\right)$ of K13A and $\mathrm{K} 27 \mathrm{~A}$ are about $15 \mathrm{mV}$ lower than those for the WT protein. In contrast, $\mathrm{K} 79 \mathrm{~A}$ displays an $E_{\mathrm{f}}$ that is $15 \mathrm{mV}$ higher compared with that of WT cyt $c$, indicating that the change in the overall net charge has no significant effect on the $E_{\mathrm{f}}$ value.

Concerning the electroactive cyt $c$ amount (surface coverage $\Gamma$ ) found for the cyt $c$ monolayer-modified gold electrodes, a rather uniform behavior has been determined with values varying between 12 and $15 \mathrm{pmol} / \mathrm{cm}^{2}$. Only the mutant K79A displays a slightly lower value. It can be concluded that the reduction in surface charge does not significantly alter the interaction with the negatively charged thiol layer on the electrode-an observation that has also been verified by SPR measurements. Here, cyt $c$ is assembled on a planar MUA/MUmodified SPR-Chip to examine the mass deposition during the adsorption process in a flow system. A clear mass deposition from low ionic strength buffer can be found for the tested proteins. The resulting concentration-dependent mass accumulation for the WT and selected protein variants is given in Figure S6. The human WT protein and the investigated 


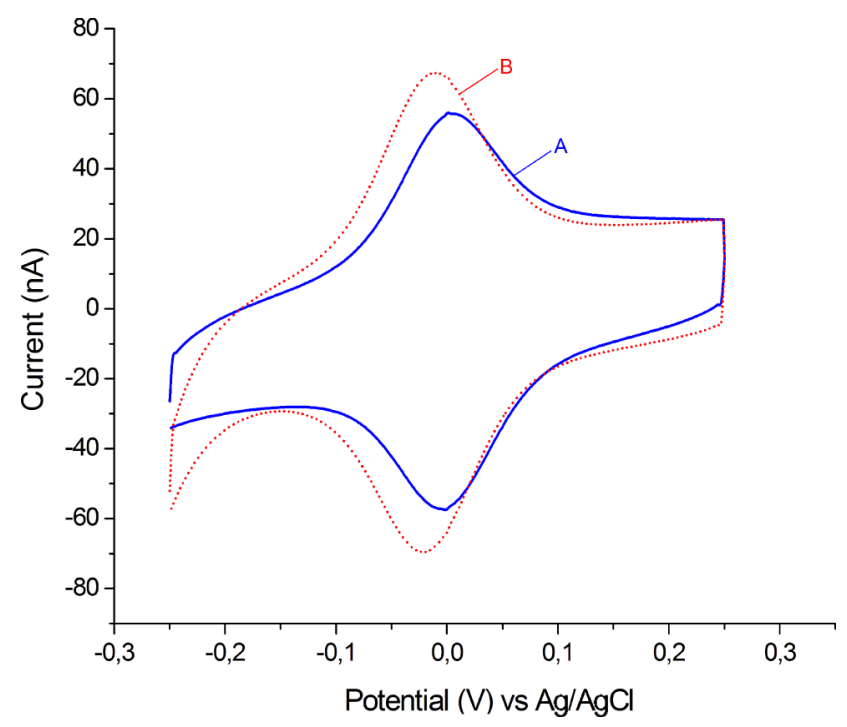

Figure 3. Cyclic voltammograms of K79A (A) and the WT (B) form of human recombinant cyt $c$ adsorbed on an MUA/MU-modified gold electrode $(5 \mathrm{mM}$ potassium phosphate buffer $\mathrm{pH} 7$ at a scan rate of $100 \mathrm{mV} / \mathrm{s})$.

mutants display rather similar mass deposition during the assembly step, confirming the data gained during the $\mathrm{CV}$ investigations.

A different behavior for some of the mutants can be observed for the $k_{\mathrm{s}}$ values. Among the Lys-substituted variants, K73A ( $k_{\mathrm{s}}$ $=95 \pm 3 \mathrm{~s}^{-1}$ ) displays a clear increase of the ET rate in comparison with that of the WT $\left(k_{\mathrm{s}}=78 \pm 6 \mathrm{~s}^{-1}\right)$. The clearly enhanced reaction rate for $\mathrm{K} 73 \mathrm{~A}$ might be due to the reduction of the positive charges close to the heme edge and thereby to an improved orientation on the electrode surface. In contrast, mutant $\mathrm{K} 27 \mathrm{~A}\left(k_{\mathrm{s}}=30 \pm 2 \mathrm{~s}^{-1}\right)$ shows a drop in the ET rate and a slightly expanded half-peak width $(105 \mathrm{mV})$, which may arise from a different docking behavior of the protein with the electrode surface

Multilayers with Different Forms of Human Cyt c. The sufficiently high rate constants for the heterogeneous ET $\left(k_{\mathrm{s}}\right)$ of the cyt $c$ variants with the electrode and the rather unaltered assembly behavior (surface coverage, $\Gamma$ ) on the negatively charged MU/MUA surface provide the basis to study the different cyt $c$ variants in a multilayer architecture. Here, SiNPs are used as a second building block to obtain defined cyt $c$ multilayers by the layer-by-layer approach. The multilayer formation is based on the electrostatic interactions among the negatively charged SiNPs (with grafted carboxylic acid groups) and the positively charged cyt $c$ and can be verified electrochemically but also by SPR and quartz crystal microbalance measurements. Electroactive multilayer electrodes have been constructed for all variants; their structure and the ET steps are schematically shown in Figure 4.

The functional properties have been analyzed by CV. Representative CVs measured for a monolayer electrode and for a four-bilayer electrode of WT cyt $c$ and SiNPs are given in Figure 5. Oxidation and reduction peaks increase with the number of layers, indicating that cyt $c$ molecules in the external layers are also electrically communicating with the electrode. Several ET steps must be considered for this electrode architecture (Figure 4): (1) the heterogeneous ET from the $\mathrm{Au}$ electrode through the SAM-layer to the cyt $c$ monolayer $\left(k_{\mathrm{s}}\right)$ and then (2) to the cyt $c$ molecules of the consecutive layers.

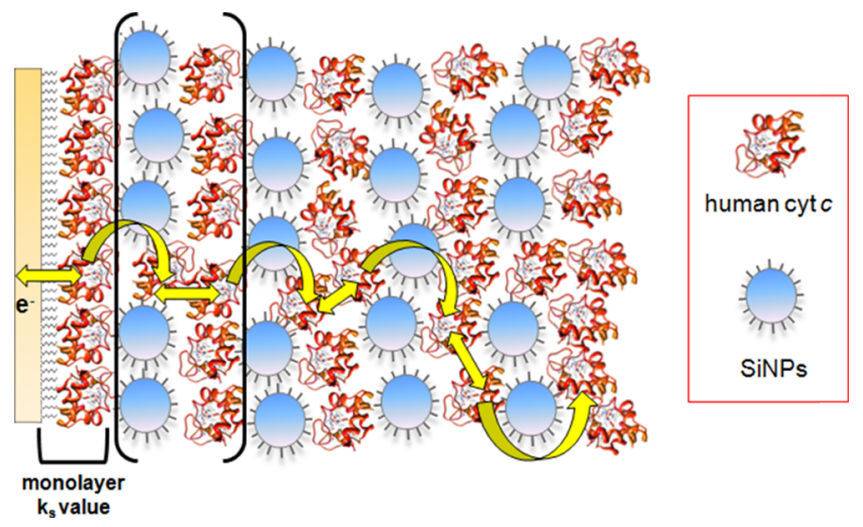

Figure 4. Schematic representation of an $\mathrm{Au}-\mathrm{MUA} / \mathrm{MU}-\mathrm{cyt}$ $c[\text { SiNPs-cyt } c]_{4}$-multilayer-coated electrode and the essential ET steps (yellow arrows).

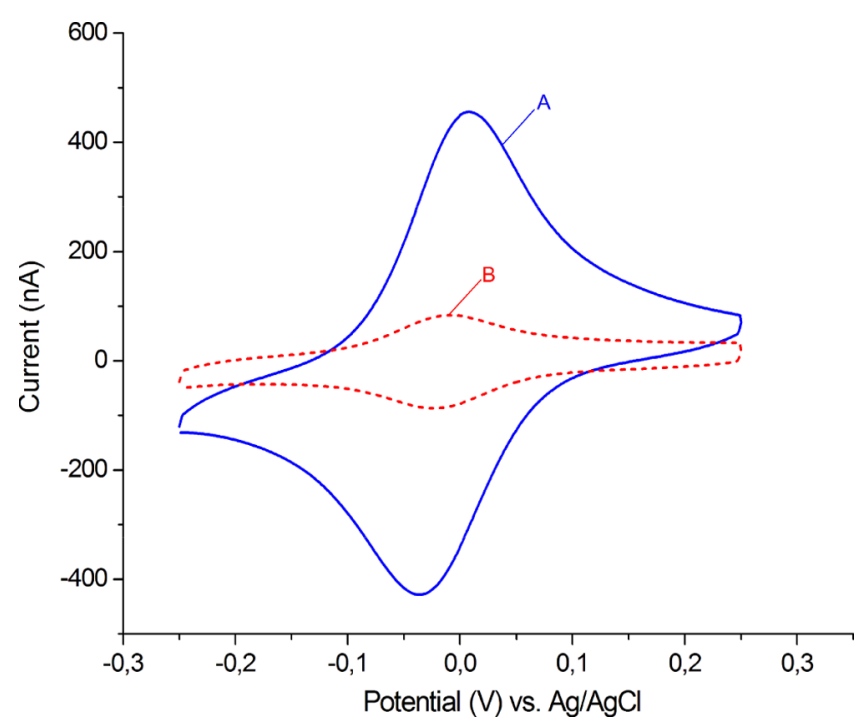

Figure 5. CVs of (B) WT cyt $c$ monolayer and (A) four-bilayer electrode, with WT cyt $c$ and SiNPs in each layer. The cyt $c$ monolayer and the four-bilayer electrode were assembled on a SAM-modified $\mathrm{Au}$ electrode. Scan rate $100 \mathrm{mV} / \mathrm{s}, 5 \mathrm{mM}$ potassium phosphate buffer, $\mathrm{pH}$ 7.0.

The adjacent steps occur by interprotein ET among the cyt $c$ molecules (self-exchange, $k_{\mathrm{ex}}$ ), thus allowing electron transport through the architecture, whereas SiNPs are just a stabilizing matrix compound, as they are nonconductive.

A closer examination of the transfer steps can be performed by a kinetic analysis with a systematic scan rate variation for all proteins in a four- and six-bilayer arrangement. The resulting CVs of four-bilayer electrodes for WT cyt $c$ and the mutants K13A, K27A, K72A, K723A, and K79A at different scan rates are shown in Figure S3, specifying the different electrochemical behavior of the cyt $c$ variants in a multilayer arrangement. For all investigated cyt $c$ electrodes, an increased peak separation with increasing scan rate can be shown, which reflects the rising kinetic limitations of the system. The formal redox potentials of the cyt $c$ variants in the multilayer architecture differ insignificantly from each other. From the analysis of the voltammetric peaks, it can be seen that the amount of electrode-addressable cyt $c$ decreases significantly at higher scan rates for all proteins. Evidently, the scan rate becomes too fast at this point, thus not all cyt $c$ molecules within the 
architecture can be converted during the period of the voltammetric scan. By quantifying the peak area (of CVs), the electroactive surface coverage $\Gamma$ values can be calculated at different scan rates and are given in Figure 6a, indicating that each cyt $c$ form has a different kinetic behavior.
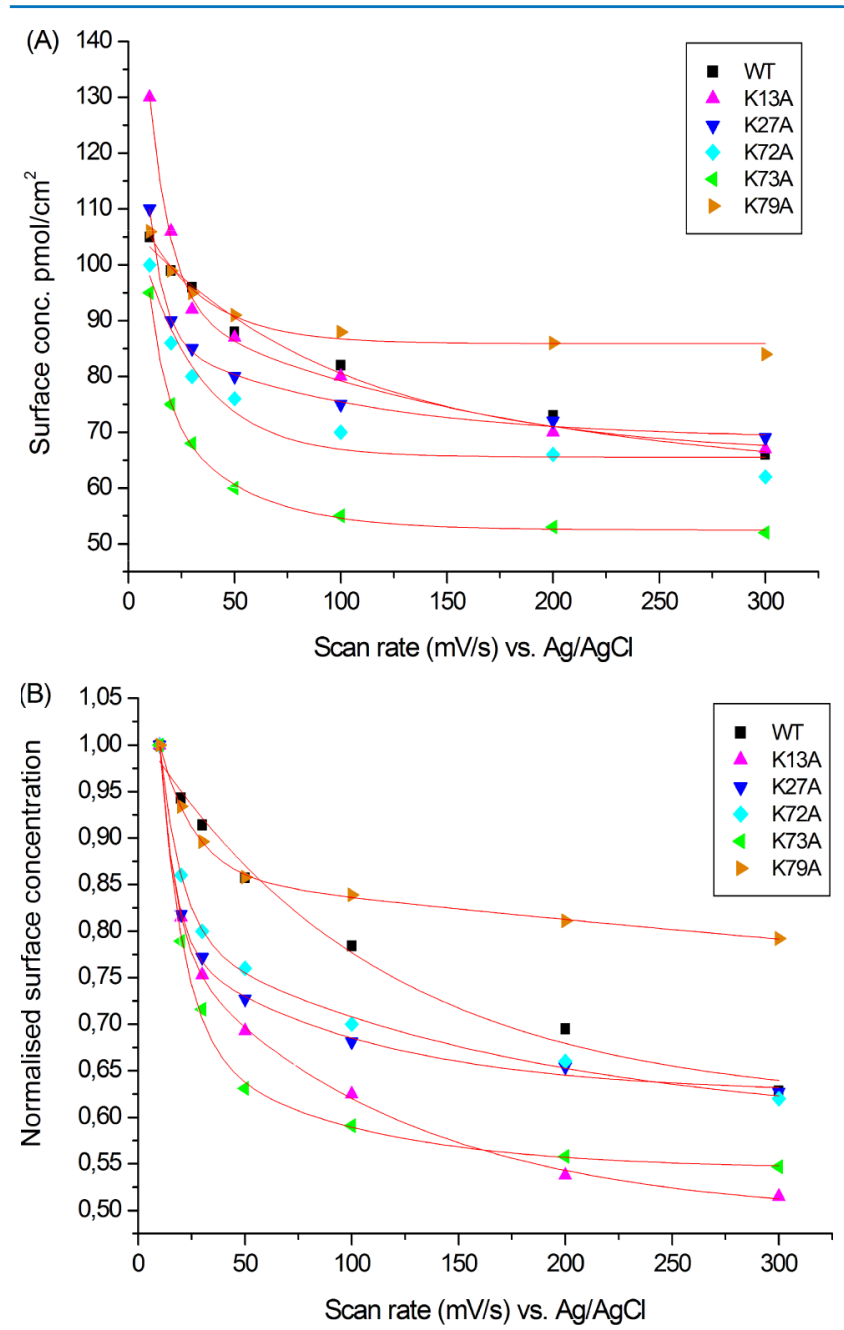

Figure 6. Electroactive surface coverage $\Gamma$ of four-bilayer electrodes with the different cyt $c$ forms as a function of different scan rates (10$300 \mathrm{mV} / \mathrm{s}$ ). Potassium phosphate buffer $5 \mathrm{mM}, \mathrm{pH}$ 7.0. (A) Absolute surface coverage $\Gamma$ for the different cyt $c$ multilayer electrodes, and (B) relative change of surface concentration by normalization to the value at $10 \mathrm{mV} / \mathrm{s}$.

The differences become even more obvious when the relative change of the electroactive amount with the scan rate is analyzed, as shown in Figure $6 \mathrm{~b}$. By analyzing the decrease in electroactive (addressable) cyt $c$, one can define a scan rate at which $80 \%$ of the starting value (at $10 \mathrm{mV} / \mathrm{s}$ ) can be voltammetrically addressed. This $\mathrm{v}_{80 \%}$ value can be seen as a qualitative tracer for the different protein behavior. Clearly, for the mutant K79A this value is larger $\left(\mathrm{v}_{80 \%}=300 \mathrm{mV} / \mathrm{s}\right)$ than it is for the WT $\left(\mathrm{v}_{80 \%}=100 \mathrm{mV} / \mathrm{s}\right)$, whereas K73A shows a much smaller value $\left(v_{80 \%}=20 \mathrm{mV} / \mathrm{s}\right)$. This suggests that the $\mathrm{K} 79 \mathrm{~A}$ mutant undergoes a more efficient interprotein electron exchange than the WT protein. Although the driving force is rapidly changed, a large portion of the cyt $c$ molecules in the multilayer system can still follow and is addressable by the electrode. In contrast, the mutation at position K73A does not show an enhanced self-exchange rate. For the K13A, K27A, and K72A mutants, a rather similar behavior as for the WT protein has been found on the electrode. By comparing the scan rate variation of the six-bilayer electrodes (Figure S4) with that of the four-bilayer ones, it can be stated that the drop in addressable cyt $c$ during the scan rate variation agrees to a large extent for the two types of multilayer electrodes. Again, the K79A mutant displays the smallest decrease in addressable cyt $c$ amount, followed by WT, K13A, K27A, and K72A; the largest decrease is exhibited by K73A.

To elucidate that the ET from the electrode to the monolayer (step 1 in Figure 4) is not the limiting step, the $k_{\text {s }}$ values of the cyt $c$ monolayer electrodes have to be taken into account. As shown in Table 2, no major decrease has been observed; rather, improved or equivalent $k_{\mathrm{s}}$ values have been found for the mutants by cyclic voltammetric investigations (except K27A displaying a lower $k_{\mathrm{s}}$ ). Thus, the different behavior in multiple layers can clearly be attributed to a variation in the self-exchange rate of the proteins $\mathrm{K} 79 \mathrm{~A}$ and K73A.

Estimation and Evaluation of Self-Exchange Rate Constants $\left(k_{\mathrm{ex}}\right)$ in Multilayer Arrangements. For further insights into the self-exchange process, the most intriguing mutants have been selected for further analysis, on the basis of their scan rate behavior (CV), reported in Figure 6. Alongside the WT protein, a mutant with a less pronounced decrease in surface coverage $\Gamma(\mathrm{K} 79 \mathrm{~A})$ and a mutant with a more pronounced decrease in $\Gamma$ (K73A) have been chosen for determination of the interprotein ET and self-exchange $\left(k_{\mathrm{ex}}\right)$ in the multilayer architecture.

To achieve an assessment of the self-exchange rate constant $\left(k_{\mathrm{ex}}\right)$ for the selected cyt $c$ variants in the investigated multilayer architectures, the Dahms-Ruff equation has been applied. ${ }^{48-50}$ Therefore, an intermolecular electron hopping process among the cyt $c$ molecules in the multilayer architecture has been assumed. The Randles-Sevcik equation ${ }^{51}$ has been used to calculate the effective diffusion coefficient, $D_{\text {effective, by }}$ evaluating the peak currents at different scan rates. For human WT cyt $c$, a $D_{\text {effective }}$ of $1.0 \times 10^{-11} \mathrm{~cm}^{2} \mathrm{~s}^{-1}$, for K73A a value of $3.8 \times 10^{-12} \mathrm{~cm}^{2} \mathrm{~s}^{-1}$, and for K79A a value of $1.3 \times$ $10^{-11} \mathrm{~cm}^{2} \mathrm{~s}^{-1}$ has been obtained. The approximate distance between the adjoining redox centers, $\delta$, is estimated to be 2.6 $\mathrm{nm}$, and for all multilayer systems an average cyt $c$ concentration of $34 \mathrm{mM}$ is used. By means of these values, subsequently, the self-exchange rate constants $\left(k_{\mathrm{ex}}\right)$ for the cyt $c$ variants are calculated: WT $2.7 \times 10^{4} \mathrm{M}^{-1} \mathrm{~s}^{-1}$, K79A $3.3 \times 10^{4}$ $\mathrm{M}^{-1} \mathrm{~s}^{-1}$, and K73A $9.9 \times 10^{3} \mathrm{M}^{-1} \mathrm{~s}^{-1}$ (see Table 3).

Table 3. Diffusion Coefficients $\left(D_{\text {eff }}\right)^{a}$ and Self-Exchange Rate Constants $\left(k_{\mathrm{ex}}\right)$ Determined in Solution (NMR) and Immobilized in a Multilayer Structure (CV) for WT Cyt $c$ and Variants

\begin{tabular}{ccccc} 
& \multicolumn{2}{c}{ immobilized $(\mathrm{CV})$} & & solution (NMR) \\
\cline { 2 - 3 } cyt $c$ forms & $D_{\text {effective }^{a, b}}{ }^{a, c}$ & $k_{\text {ex }}$ & $k_{\text {ex }}$ \\
WT & $1.0 \times 10^{-11}$ & $2.7 \times 10^{4}$ & & $5.6 \times 10^{3}$ \\
K73A & $3.8 \times 10^{-12}$ & $9.9 \times 10^{3}$ & & $3.3 \times 10^{3}$ \\
K79A & $1.3 \times 10^{-11}$ & $3.3 \times 10^{4}$ & & $5.9 \times 10^{3}$
\end{tabular}

${ }^{a}$ All data are evaluated as the average values of at least three electrodes. ${ }^{b} D_{\text {effective }}$ is calculated according to the Randles-Sevcik equation and given as $\mathrm{cm}^{2} \mathrm{~s}^{-1} .{ }^{c} k_{\mathrm{ex}}$ is calculated according to the Dahms-Ruff equation and given as $\mathrm{M}^{-1} \mathrm{~s}^{-1}$. 
It needs to be mentioned that the applied model is based on the idea of hopping electrons among the heme sites of the adjacent cyt $c$ molecules, and not assuming diffusing cyt $c$ molecules, as the cyt $c$ molecules are immobilized in the multilayer architecture. The observed changes in $k_{\text {ex }}$ are defined in the following order of efficiency of the self-exchange reaction: $\mathrm{K} 79 \mathrm{~A}>\mathrm{WT} \gg \mathrm{K} 73 \mathrm{~A}$. This is also in good agreement with the qualitative analysis obtained by the $\mathrm{v}_{80 \%}$ values.

Despite the presumed high ionic strength ${ }^{52}$ inside the multilayer architecture owing to the negatively charged SiNPs, the calculated values appear rather high. This might be explained by the high amount of protein deposited on the electrode and by the residual rotational flexibility of the cyt $c$ molecules inside the multilayers. In addition, the local cyt $c$ concentration may also be affected by the specific cyt $c$ variant embedded in the layered architecture. Given that a precise determination is not feasible, an equal cyt $c$ concentration within the investigated protein multilayer electrodes has been assumed for our calculations.

The increased self-exchange rate for cyt $c$ mutant K79A and the decreased self-exchange rate for K73A in comparison with that of the WT protein demonstrate the presence of key protein-protein interactions that modulate the ET process and thus the $k_{\text {ex }}$. The different location of the mutated Lys residues on the protein surface provides hints about the position of hot spots for the formation of productive protein-protein complexes. As a consequence, the analyzed variants modulate the protein recognition process unequally, from the unaltered effect induced by K13A, K27A, and K72A to the significant changes of opposite sign for K79A and K73A.

NMR Measurement of Self-Exchange Rate Constants $\left(k_{\mathrm{ex}}\right)$ in Solution. Electron self-exchange rates in solution have also been evaluated by NMR for three selected proteins (WT, $\mathrm{K} 73 \mathrm{~A}$, and $\mathrm{K} 79 \mathrm{~A})$. The ${ }^{1} \mathrm{H}-{ }^{15} \mathrm{~N}$ HSQC spectra obtained on samples with almost equimolar mixtures of the two redox states contain two $\mathrm{HN}$ amide peaks $\left(\mathrm{N}_{\mathrm{ox}} \mathrm{H}_{\mathrm{ox}}\right.$ and $\left.\mathrm{N}_{\text {red }} \mathrm{H}_{\text {red }}\right)$ for each residue, as shown in Figure S5, top panel. In the ${ }^{1} \mathrm{H}-{ }^{15} \mathrm{~N}$ HSQC- ${ }^{15} \mathrm{~N}$ exchange spectroscopy (EXSY) experiment, for each amide group two additional peaks are detected, that is, the exchange peaks $\mathrm{N}_{\text {ox }} \mathrm{H}_{\text {red }}$ and $\mathrm{N}_{\text {red }} \mathrm{H}_{\text {ox }}$. (Figure S5, bottom panels). Using molar fractions and peak intensities, a qualitative analysis is possible, as described in the Materials and Methods section, and self-exchange rate constants for the WT cyt $c$ and its variants (K73A, K79A) can be determined. The $k_{\mathrm{ex}}$ values in solution measured by NMR are smaller than those measured in the multilayer structure. However, their relative values follow the same order of efficiency of the self-exchange reaction: K79A $>\mathrm{WT} \gg \mathrm{K} 73 \mathrm{~A}$. The $k_{\text {ex }}$ values obtained in solution and in multilayer arrangements are summarized in Table 3.

The two variants K79A and K73A are reasonably affected to a similar extent upon replacement of a surface Lys with an Ala, when considering long-range electrostatic effects. At variance, both methodologies identify an increased $k_{\mathrm{ex}}$ (with respect to WT) for K79A and a decreased one for K73A, which should be driven by local contacts.

The protein-protein contacts between the cyt $c$ molecules that give rise to electron self-exchange are not known. A reasonable model for what happens in the immobilized state could be provided by the molecular contacts observed in the crystal structure of human cyt $c$ (PDB 3ZCF), which contains four molecules in the unit cell. ${ }^{53}$ Although a crystal structure cannot verify ET pathways, it can give valuable hints. Interestingly, recent studies have shown that in calixarene- supported cyt $c$ crystals ET between fixed redox sites is feasible. $^{54}$

It is apparent from the above-mentioned crystal structure that $\mathrm{K} 79 \mathrm{~A}$ and $\mathrm{K} 73 \mathrm{~A}$ are both located in the interaction surface area. K73A displays a high heterogeneous ET rate but a reduced self-exchange rate. Therefore it seems reasonable to propose that altering the nature of the amino acid at this position hinders the ability of the protein to give rise to selfexchange reactions. The differences in $k_{\mathrm{s}}$ and $k_{\text {ex }}$ between K79A and WT are less pronounced but point to a decreased $k_{\mathrm{s}}$ and increased $k_{\mathrm{ex}}$, suggesting that the replacement with an Ala at this position facilitates the formation of a productive selfexchange ET complex.

This study shows that there is no simple correlation between the heterogeneous ET rate of cyt $c$ at a thiol-modified gold electrode and the self-exchange properties of cyt $c$ within a complex multilayer system. This is not only the case for the K79A and K73A variants but also for K27A, wherein unaltered exchange behavior is found in multiple layers, but a decreased reaction rate is observed at the electrode. Furthermore, the correlation between the cyt $c$ self-exchange behavior in solution and in a multilayer assembly points to a comparable situation for the investigated proteins in these situations and may support the idea of rotational flexibility of the variants in the adsorbed state.

\section{CONCLUSIONS}

Different variants of human cyt $c$ have been examined regarding their heterogeneous ET rate constant $\left(k_{\mathrm{s}}\right)$ adsorbed in a monolayer state on a thiol-modified gold electrode and their self-exchange rate constant $\left(k_{\mathrm{ex}}\right)$ incorporated in a multilayer assembly. The latter has importance as a basic platform to integrate and connect different enzymes with electrodes.

For this purpose, five mutants of the human WT protein have been chosen with a focus on their surface electrostatic properties by replacing a lysine residue with an alanine (K13A, K27A, K72A, K73A, and K79A). It has been shown that singlepoint mutations in the human forms of cyt $c$ can significantly influence the heterogeneous ET $\left(k_{\mathrm{s}}\right)$ with MU/MUA-modified gold electrodes. Here, K73A results in higher and K27A in lower $k_{\mathrm{s}}$ values in comparison with those of the human WT cyt $c$ and the other protein variants.

It is further demonstrated that electroactive protein multilayers can be constructed with all mutant forms of human cyt $c$ investigated here. The amount of immobilized protein by the layer-by-layer approach is found to be not highly dependent on the protein variant used. However, the kinetic behavior of the voltammetric signals is different. Whereas the K79A mutant shows an enhanced electron exchange rate compared with that of the WT (and K13A, K27A, and K72A), K73A displays a reduced self-exchange. This behavior has been verified with multilayer electrodes with different number of layers. The same trend $(\mathrm{K} 79 \mathrm{~A}>\mathrm{WT}>\mathrm{K} 73 \mathrm{~A})$ has been observed by measuring self-exchange rates in solution by NMR. The K79A variant, which has the largest increase in $k_{\text {ex }}$ and the largest formal redox potential, is the one with the mutation site closer to the heme. These experiments provide emerging evidence that the simple reduction in surface charge is not the only determinant for an altered self-exchange of the variants and that the specific location of the residues on the protein surface drives the process.

Altogether, three different determinants can be specified that affect the electrochemical properties of cyt $c$ in a redox-active 
multilayer architecture: (1) the amount of electrochemically addressable cyt $c$ molecules per layer that can be deposited on the electrode, (2) the cyt $c$ self-exchange rate $\left(k_{\text {ex }}\right)$, and (3) the heterogeneous rate constant $\left(k_{\mathrm{s}}\right)$ for the ET with the electrode. The study demonstrates that cyt $c$ self-exchange can be a valuable tool for constructing complex and functional protein electrodes. It shows that the application of a protein variant with an enhanced self-exchange rate can improve the kinetic behavior of cyt $c$-based multilayer architectures allowing a faster electron transport through multiple protein layers on electrodes. This may also have prospects for an improved connection of enzymes to electrodes through multiple layers of cyt $c$.

\section{MATERIALS AND METHODS}

Materials. Potassium hydroxide (Sigma-Aldrich, Taufkirchen, Germany), potassium dihydrogen phosphate $\left(\mathrm{KH}_{2} \mathrm{PO}_{4}\right)$, dipotassium hydrogen phosphate $\left(\mathrm{K}_{2} \mathrm{HPO}_{4}\right)$, potassium chloride (Merck, Darmstadt, Germany), gold-wire electrodes, diameter of $0.5 \mathrm{~mm}$ (Goodfellow, Bad Nauheim, Germany), 11-mercapto-1-undecanoic acid, 11-mercapto-1-undecanol (Sigma-Aldrich, Taufkirchen, Germany).

Buffers. $\mathrm{K}_{2} \mathrm{HPO}_{4}$ and $\mathrm{KH}_{2} \mathrm{PO}_{4}$ were used for preparation of the potassium phosphate buffer $(5 \mathrm{mM}, \mathrm{pH} 7.0)$; the $\mathrm{pH}$ was adjusted with phosphoric acid or potassium hydroxide.

SiNPs. The SiNPs were synthesized and characterized according to a well-established synthesis protocol. ${ }^{24}$

Protein Mutant Preparation (Mutation of Human Cyt c). The pET21a-CCHLhCYC plasmid (for the human cyt $c$ gene) was provided by Chuang. ${ }^{55}$ The pET21a-CCHLhCYC plasmid was used to introduce mutations (referred to as WT). The Quick Change site-directed mutagenesis kit (Stratagene, La Jolla) was used to introduce mutations according to the provided protocol. The mutations were confirmed by gene sequencing.

Purification/Expression of Recombinant WT and Mutated Human Cyt c. For the purification and expression of human cyt $c$, previous protocols were adapted. ${ }^{55,56}$ Human cyt $c$ was coexpressed with cyt $c$ maturation and hemelyase. Transformation of the plasmid DNA (mutated cyt $c$ gene) was realized by the use of BL21- (DE3)C41 Escherichia coli cells. ${ }^{57}$ Cultivation of the these cells was carried out in minimal M9 media (addition of vitamins, minerals, and glycerol) at $37^{\circ} \mathrm{C}$, $180 \mathrm{rpm}$. Expression was initialized by addition of $\mathrm{FeSO}_{4}(100$ $\mathrm{mg} / \mathrm{L})$ and IPTG $(1 \mathrm{mM})$. The culture was incubated at $30^{\circ} \mathrm{C}$ $(60 \mathrm{rpm})$ for $72 \mathrm{~h}$. The cells were harvested by centrifugation and lysated with lysozyme and sonication. Two chromatography steps were needed to purify cyt $c$. In the first step, the supernatant (lysate) was separated by the use of an SP Sepharose cationic exchange column (GE Healthcare, Sweden). The protein was eluted with $\mathrm{NaCl}$ (linear gradient, $0-500 \mathrm{mM}$, in sodium phosphate buffer $(50 \mathrm{mM}), \mathrm{pH}$ 6.8). Sodium dodecyl sulfate-polyacrylamide gel electrophoresis was applied to analyze the collected protein fractions. The ones containing cyt $c$ were further used, and an ultracentrifugal filter $\left(M_{\mathrm{w}}\right.$ cutoff of $5000 \mathrm{kDa}$; Amicon; Millipore) was used to concentrate them. Afterward, the concentrate sample was further purified with: Hi Load 16/60 Superdex 75 prep grade column (GE Healthcare, Sweden), sodium phosphate buffer (50 mM), $\mathrm{pH}$ 6.8 .

Spectrophotometric Investigations. Spectrophotometric measurements were carried out with a UV-vis spectrophotometer (UV-250 1PC, Shimadzu Scientific Instruments, Kyoto, Japan). Spectrums were recorded between 350 and
$650 \mathrm{~nm}$ (cyt $c$ conc. $5 \mu \mathrm{M}$ ). For oxidizing cyt $c$, ferricyanide was used, and for reducing cyt $c$, dithiothreitol (DTT) was used. The protein samples were also dialyzed $(50 \mathrm{mM}$ sodium phosphate, $\mathrm{pH}$ 7.5, Slide-A-Lyzer dialyses cassettes; Thermo Scientific, Rockford, IL, $M_{\mathrm{w}}$ cutoff $=3500 \mathrm{kDa}$ ).

SPR Experiments. To investigate the adsorption process of the cyt $c$ variants on a SAM-modified Au electrode, Biacore X and T100 (Biacore AB, Sweden) were used. The SPR-Au chip was cleaned according to an already established protocol. Briefly, the Au chip was incubated for 10 min in a $\mathrm{H}_{2} \mathrm{SO}_{4} / \mathrm{H}_{2} \mathrm{O}_{2}$ (3:1) solution. Afterward, the SAM-layer formation was initiated by incubation of the Au chip for $48 \mathrm{~h}$ in a MUA/ MU-solution (1:3; $5 \mathrm{mM})$. Cyt $c$ solutions were flushed over the SAM-modified chip with a flow rate of $1 \mu \mathrm{L} / \mathrm{min}$.

NMR Experiments. Unlabeled and ${ }^{15} \mathrm{~N}$-labeled cyt $c$ were used for NMR studies, in its paramagnetic ferric and diamagnetic ferrous form. For oxidizing cyt $c$, ferricyanide was used, and for reducing cyt $c$ DTT was used. By means of ultrafiltration, the oxidizing agent was removed ahead of NMR measurements. The protein samples $(0.7 \mathrm{mM})$ were concentrated with a Centricon centrifugal filter $\left(M_{\mathrm{w}}\right.$ cutoff $=5000$ $\mathrm{Da})$, phosphate buffer $(50 \mathrm{mM}), \mathrm{pH} 6.8,10 \% \mathrm{D}_{2} \mathrm{O}$. For the electron self-exchange measurements, the required oxidized/ reduced ratio was preserved by addition of DTT in stoichiometric amounts. A Bruker Avance Spectrometer (16.4 T, $700 \mathrm{MHz}$ ) was used for the NMR investigations. Ferric cyt $c$ undergoes autoreduction of the heme iron in solution. ${ }^{58}$ To maintain the selected oxidized/reduced ratio through the experiments, electron self-exchange measurements were conducted at $289 \mathrm{~K}$. Four sets of experiments were recorded for each sample. For detection of the diamagnetic part, the $1 \mathrm{D}{ }^{1} \mathrm{H}$ NMR spectrum was recorded (spectral width $=20 \mathrm{ppm}, 2.0 \mathrm{~s}$ recycle delay). To detect the hyperfine-shifted signals in the ferric form (cyt $c$ variants) a spectral width of $100 \mathrm{ppm}$ was used in combination with faster repetition rates of $800 \mathrm{~ms}$. Two-dimensional (2D) ${ }^{1} \mathrm{H}-{ }^{15} \mathrm{~N}$ HSQC experiments were performed with $2048 \times 200$ data points, a spectral width of $15 \mathrm{ppm}$ for ${ }^{1} \mathrm{H}$, and $40 \mathrm{ppm}$ for ${ }^{15} \mathrm{~N}$. Self-exchange rates were measured with the ${ }^{1} \mathrm{H}-{ }^{15} \mathrm{~N}$ HSQC $-{ }^{15} \mathrm{~N}$ EXSY pulse sequence. ${ }^{33}$ For calculation of the self-exchange rate constants, the following equation was used

$$
\begin{aligned}
& \sqrt{\frac{V_{\text {red-ox }} V_{\text {ox-red }}}{V_{\text {ox }} V_{\text {red }}}} \\
& =\sqrt{\frac{f_{\text {ox }}^{2} f_{\text {red }}^{2}\left(1-\mathrm{e}^{-k_{\text {ex }} \tau_{\mathrm{m}}}\right)^{2}}{\left(f_{\text {red }}^{2}+f_{\text {ox }} f_{\text {red }} \mathrm{e}^{-k_{\text {ex }} \tau_{\mathrm{m}}}\right)\left(f_{\text {ox }}^{2}+f_{\text {ox }} f_{\text {red }} \mathrm{e}^{\left.-k_{\text {ex }} \tau_{\mathrm{m}}\right)}\right.}}
\end{aligned}
$$

In this equation, $V_{\text {ox }}$ and $V_{\text {red }}$ are the volumes of oxidized and reduced self-peaks, and $V_{\text {red-ox }}$ and $V_{\text {ox-red }}$ are the volumes of the two connecting exchange peaks; their values are derived upon integration of resolved sets of signals in the ${ }^{1} \mathrm{H}-{ }^{15} \mathrm{~N}$ HSQC $-{ }^{15} \mathrm{~N}$ EXSY. The molar ratios of oxidized and reduced cyt $c$ forms ( $f_{\text {ox }}$ and $f_{\text {red }}$, respectively) were measured from the relative intensity of peaks that correspond to the same amino acid in the two redox forms of the protein in the ${ }^{1} \mathrm{H}-{ }^{15} \mathrm{~N}$ HSQC maps. $\tau_{\mathrm{m}}$ is the mixing time in the EXSY experiment. The self-exchange rate constant, $k_{\mathrm{ex}}$, is the only parameter to be fitted and can be calculated from the above equation.

Construction of the Cyt $c$ Mono- and Multilayer Electrodes. The gold-wire electrodes were cleaned and SAMmodified according a well-established protocol. ${ }^{28}$ Cyt $c$ 
monolayer and multilayer electrodes were prepared according an already published protocol. ${ }^{24}$

Electrochemical Investigations. Electrochemical measurements were performed with a $\mathrm{CHI}-660 \mathrm{E}$ potentiostat $(\mathrm{CHI}$ Instruments, Austin, TX). A custom-made cell ( $1 \mathrm{~mL}$ ) was used for the electrochemical investigations. A platinum electrode was used as counter electrode, and as reference electrode an $\mathrm{Ag} /$ $\mathrm{AgCl}(1 \mathrm{M} \mathrm{KCl})$ with a potential of $+0.237 \mathrm{~V}$ versus $\mathrm{NHE}$ (Biometra, Göttingen, Germany) was used. The electrochemical properties of the cyt $c$ mono- and multilayer electrodes by $\mathrm{CV}$ were recorded between -350 and +350 $\mathrm{mV}$ ( vs Ag/AgCl; scan rate $=100 \mathrm{mV} / \mathrm{s}$ ). A scan rate variation (between $10 \mathrm{mV} / \mathrm{s}$ and $15 \mathrm{~V} / \mathrm{s}$ ) was performed to assess the heterogeneous ET rate constant, $k_{s}$, for the cyt $c$ monolayer electrodes. The resulting peak separation was evaluated by applying the Laviron method. ${ }^{59}$ The self-exchange rate constants, $k_{\mathrm{ex}}$ for the multilayer electrodes were assessed by a scan rate variation $(10 \mathrm{mV} / \mathrm{s}$ to $15 \mathrm{~V} / \mathrm{s})$ and evaluated according to the Dahms-Ruff equation. ${ }^{48-50}$ The RandlesSevcik equation ${ }^{51}$ was used to calculate (peak currents at different scan rates) the diffusion coefficients. The redox-active cyt $c$ surface coverage $(\Gamma)$ was analyzed (applying Faraday's law) by using the average of the peak area (oxidation and reduction charge). Half-peak width and charge were evaluated with the CHI software.

\section{ASSOCIATED CONTENT}

\section{S Supporting Information}

The Supporting Information is available free of charge on the ACS Publications website at DOI: 10.1021/acsomega.6b00213.

UV-vis spectra, cyclic voltammograms, 2D NMR, and SPR data (PDF)

\section{AUTHOR INFORMATION}

\section{Corresponding Authors}

*E-mail: feifel@th-wildau.de (S.C.F.). Phone: +49(0)3375508158.

*E-mail: flisdat@th-wildau.de (F.L.).

\section{ORCID}

Sven Christian Feifel: 0000-0001-6296-5236

\section{Author Contributions}

The electrode construction and electrochemical characterization were carried out by S.C.F. The SPR experiments were performed by K.R.S. The electrochemical and SPR data were evaluated by S.C.F., A.K., K.R.S., and F.L. Cyt $c$ mutants were prepared by D.W. and provided by P.T. NMR measurements were carried by M.A. and M.P. The NMR data were evaluated by M.A., M.P., and P.T. The concept was initiated by F.L. and P.T. The manuscript was written through contributions of all authors. All authors have given approval to the final version.

\section{Notes}

The authors declare no competing financial interest.

\section{ACKNOWLEDGMENTS}

The authors thank the Deutsche Forschungsgemeinschaft (grant DFG Li 706/7-1). This work was supported by Instruct, part of the European Strategy Forum on Research Infrastructures (ESFRI) and supported by national member subscriptions. We thank the EU-ESFRI Instruct Core Center CERM, Italy, for the NMR and protein expression facilities.

\section{REFERENCES}

(1) Katz, A. E.; Willner, I. Integrated nanoparticle-biomolecule hybrid systems: synthesis, properties, and applications. Angew. Chem., Int. Ed. 2004, 43, 6042-6108.

(2) Willner, I.; Katz, E. Integration of Layered Redox Proteins and Conductive Supports for Bioelectronic Applications. Angew. Chem., Int. Ed. 2000, 39, 1180-1218.

(3) Yu, X.; Chattopadhyay, D.; Galeska, I.; Papadimitrakopoulos, F.; Rusling, J. F. Peroxidas activity of enzymes bound to the ends of single-wall carbon nanotube forest electrodes. Electrochem. Commun. 2003, 5, 408-411.

(4) Fan, C. H.; Suzuki, I.; Chen, Q.; Li, G. X.; Anzai, J. An unmediated hydrogen peroxide sensor based on hemoglobin-SDS film modified electrode. Anal. Lett. 2000, 33, 2631-2644.

(5) Wegerich, F.; Turano, P.; Allegrozzi, M.; Möhwald, H.; Lisdat, F. Cytochrome $c$ mutants for superoxide biosensors. Anal. Chem. 2009, 81, 2976-2984.

(6) Ganesana, M.; Erlichman, J. S.; Andreescu, S. Real-time monitoring of superoxide accumulation and antioxidant activity in brain slice model using an electrochemical cytochrome $c$ biosensor. Free Radical Biol. Med. 2012, 53, 2240-2249.

(7) Shleev, S.; Wettero, J.; Magnusson, K. E.; Ruzgas, T. Electrochemical characterization and application of azurin-modified gold electrodes for detection of superoxide. Biosens. Bioelectron. 2006, 22, 213-219.

(8) Lisdat, F.; Ge, B.; Stöcklein, W.; Scheller, F. W.; Meyer, T. Electrochemical Behavior and Nitric Oxide Integration of Immobilized Cytochrome $\mathrm{c}^{\prime}$ from Rhodocyclusgelantinosus. Electroanalysis 2000, 12, 946-951.

(9) Scott, R. A.; Mauk, A. G. Cytochrome c: A Multidisciplinary Approach; University Science Books: Sausalito, CA, 1995; pp 192197.

(10) Fedurco, M. Redox reactions of heme-containing metalloproteins: dynamic effects of self-assembled monolayers on thermodynamics and kinetics of cytochrome $c$ electron transfer reactions. Coord. Chem. Rev. 2000, 209, 263-269.

(11) Murgida, D. H.; Hildebrandt, P. Surface-enhanced vibrational spectroelectrochemistry: electric-field effects on redox and redoxcoupled processes of heme proteins, in surface-enhanced Raman scattering: physics and applications. Top. Appl. Phys. 2006, 103, 313334.

(12) Murgida, D. H.; Hildebrandt, P. Redox and redox-coupled processes of heme proteins and enzymes at electrochemical interfaces. Phys. Chem. Chem. Phys. 2005, 7, 3773-3784.

(13) Murgida, D. H.; Hildebrandt, P. Electron transfer processes of cytochrome $c$ at interfaces. New insights by surface-enhanced resonance Raman spectroscopy. Acc. Chem. Res. 2004, 37, 854-861.

(14) Nakano, K.; Yoshitake, T.; Yamashita, Y.; Bowden, E. F. Cytochrome $c$ self-assembly on alkanethiol monolayer electrodes as characterized by AFM, IR, QCM, and direct electrochemistry. Langmuir 2007, 23, 6270-6275.

(15) Lisdat, F.; Ge, B.; Ehrentreich-Forster, E.; Reszka, R.; Scheller, F. W. Superoxide dismutase activity measurement using cytochrome $c$ modified electrode. Anal. Chem. 1999, 71, 1359-1365.

(16) Cooper, J. M.; Greenough, K. R.; McNeil, C. J. Direct electron transfer between immobilized cytochrome $c$ and modified gold electrodes. J. Electroanal. Chem. 1993, 347, 267-275.

(17) Wegerich, F.; Turano, P.; Allegrozzi, M.; Möhwald, H.; Lisdat, F. Electroactive multilayer assemblies of bilirubin oxidase and human cytochrome $c$ mutants: insights in formation and kinetic behavior. Langmuir 2011, 27, 4202-4211.

(18) Büttemeyer, R.; Philipp, A. W.; Mall, J. W.; Ge, B.; Scheller, F. W.; Lisdat, F. In vivo measurement of oxygen-derived free radicals during reperfusion injury. Microsurgery 2002, 22, 108-113.

(19) Lisdat, F.; Ge, B.; Reszka, R.; Kozniewska, E. An electrochemical method for quantification of the radical scavenging activity of SOD. Fresenius' J. Anal. Chem. 1999, 365, 494-498. 
(20) Ge, B.; Lisdat, F. Superoxide sensor based on cytochrome c immobilized on mixed-thiol SAM with a new calibration method. Anal. Chim. Acta 2002, 454, 53-64.

(21) Beissenhirtz, M. K.; Scheller, F. W.; Stocklein, W. F. M.; Kurth, D. G.; Mohwald, H.; Lisdat, F. Electroactive cytochrome $c$ multilayers within a polyelectrolyte assembly. Angew. Chem., Int. Ed. 2004, 43, 4357-4360.

(22) Sarauli, D.; Tanne, J.; Schäfer, D.; Schubart, I. W.; Lisdat, F. Multilayer Electrodes: Fully Electroactive Cyt $c$ on Gold as Part of a DNA/protein Architecture. Electrochem. Commun. 2009, 11, 22882291.

(23) Bonk, S. M.; Lisdat, F. Layer-by-Layer Assembly of Electroactive Gold-Nanoparticle/Cytochrome c Multilayers. Biosens. Bioelectron. 2009, 25, 739-744.

(24) Feifel, S. C.; Lisdat, F. Silica Nanoparticles for the Layer-byLayer Assembly of Fully Electro-active Cytochrome $c$ Multilayer. J. Nanobiotechnol. 2011, 9, 59.

(25) Ma, H.; Hu, N.; Rusling, J. F. Electroactive Myoglobin films grown layer-by-layer with poly(styrenesulfonate) on pyrolytic graphite electrodes. Langmuir 2000, 16, 4969-4975.

(26) Beissenhirtz, M. K.; Scheller, F. W.; Lisdat, F. A superoxide sensor based on a multilayer cytochrome $c$ electrode. Anal. Chem. 2004, 76, 4665-4671.

(27) Scott, R.; Mauk, G. Cytochrome C: A Multidisciplinary Approach; University Science Books: Sausalito, CA, 1996; pp 643-646.

(28) Feifel, S. C.; Kapp, A.; Ludwig, R.; Lisdat, F. Nanobiomolecularmultiprotein clusters on electrodes for formation of a switchable cascadic reaction scheme. Angew. Chem., Int. Ed. 2014, 53, $5676-5679$.

(29) Dronov, R. V.; Kurth, D. G.; Möhwlad, H.; Scheller, F. W.; Lisdat, F. Layer-by-Layer Arrangement by Protein-Protein Interaction of Sulfite Oxidase and Cytochrome $c$ Catalyzing Oxidation of Sulfite. J. Am. Chem. Soc. 2008, 130, 1122-1123.

(30) Stieger, K. R.; Ciornii, D.; Kölsch, A.; Hejazi, M.; Lokstein, H.; Feifel, S. C.; Zouni, A.; Lisdat, F. Engineering of supramolecular photoactive protein architectures: the defined co-assembly of photosystem I and cytochrome $c$ using a nanoscaled DNA-matrix. Nanoscale 2016, 8, 10695-10705.

(31) Gupta, R. K.; Redfield, A. G. Double nuclear magnetic resonance observation of electron exchange between ferri- and ferro cytochrome c. Science 1970, 169, 1204-1206.

(32) Santos, H.; Turner, D. L.; Xavier, A. V.; LeGall, J. Twodimensional NMR studies of electron transfer in cytochrome c3. J. Magn. Reson. 1984, 59, 177-180.

(33) Ubbink, M.; Canters, G. W. Mutagenesis of the conserved lysine 14 of cytochrome c-550 from Thiobacillusversutus affects the protein structure and the electron self-exchange rate. Biochemistry 1993, 32, 13893-13901.

(34) Bertini, I.; Gaudemer, A.; Luchinat, C.; Piccioli, M. Electron selfexchange in high potential iron-sulfur proteins. Characterization of protein I from Ectothiorhodospiravacuolata. Biochemistry 1993, 32, 12887-12893.

(35) Cacciatore, S.; Piccioli, M.; Turano, P. Electron self-exchange of cytochrome $c$ measured via ${ }^{13} \mathrm{C}$ detected proton-less NMR. $J$. Porphyrins Phthalocyanines 2013, 17, 142-149.

(36) Döpner, S.; Hildebrandt, P.; Rosell, F. I.; Mauk, A. G.; Walter, M.; von Buse, G.; Soulimane, T. The structural and functional role of lysine residues in the binding domain of cytochrome $c$ in the electron transfer to cytochrome c oxidase. Eur. J. Biochem. 1999, 261, 379-391.

(37) Wegerich, F.; Giachetti, A.; Allegrozzi, M.; Lisdat, F.; Turano, P. Mechanistic insights into the superoxide-cytchrome $c$ reaction by lysine surface scanning. J. Biol. Inorg. Chem. 2013, 18, 429-440.

(38) Meyer, T. E.; Kamen, M. D. New perspectives on c-type cytochromes. Adv. Protein Chem. 1982, 35, 105-212.

(39) Concar, D. W.; Hill, H. A. O.; Moore, G. R.; Whitford, D.; Williams, R. J. P. The modulation of cytochrome $c$ electron selfexchange by site-specific chemical modification and anion binding. FEBS Lett. 1986, 206, 15-19.
(40) Bortolotti, C. A.; Siwko, M. E.; Castellini, E.; Ranieri, A.; Sola, M.; Corni, S. The reorganization energy in cytochrome $c$ is controlled by the accessibility of the heme to the solvent. J. Phys. Chem. Lett. 2011, 2, 1761-1765.

(41) Schreiber, G.; Haran, G.; Zhou, H. X. Fundamental aspects of protein-protein association kinetics. Chem. Rev. 2009, 109, 839-860.

(42) Bashir, Q.; Scanu, S.; Ubbink, M. Dynamics in electron transfer protein complexes. FEBS J. 2011, 278, 1391-400.

(43) Ubbink, M. The courtship of proteins: understanding the encounter complex. FEBS Lett. 2009, 583, 1060-1066.

(44) Bertini, I.; Cavallaro, G.; Rosato, A. A structural model for the adduct between cytochrome $c$ and cytochrome $c$ oxidase. J. Biol. Inorg. Chem. 2005, 10, 613-624.

(45) Schmidt, T. R.; Wildman, D. E.; Uddin, M.; Opazo, J. C.; Goodman, M.; Grossman, L. I. Rapid electrostatic evolution at the binding site for cytochrome $c$ on cytochrome $c$ oxidase in anthropoid primates. Proc. Natl. Acad. Sci. U.S.A. 2005, 102, 6379-6384.

(46) Bertini, I.; Turano, P.; Vila, A. J. Nuclear magnetic resonance of paramagnetic metalloproteins. Chem. Rev. 1993, 93, 2833-2932.

(47) Assfalg, M.; Banci, L.; Bertini, I.; Bruschi, M.; Turano, P. 800 $\mathrm{MHz} 1 \mathrm{H}$ NMR solution structure refinement of oxidized cytochrome c7 from Desulfuromonasacetoxidans. Eur. J. Biochem. 1998, 256, 261270.

(48) McKenzie, K. J.; Marken, F. Accumulation and reactivity of the redox protein cytochrome $c$ in mesoporous films of $\mathrm{TiO}_{2}$ phytate. Langmuir 2003, 19, 4327-4331.

(49) Ruff, I.; Friedrich, V. J. Transfer Diffusion. I. Theoretical. J. Phys. Chem. 1971, 75, 3297-3302.

(50) Dahms, H. Electronic conduction in aqueous solution. J. Phys. Chem. 1968, 72, 362-364.

(51) Bard, A. J.; Faulkner, L. R. Electrochemical Methods, Fundamentals and Applications; John Wiley and Sons: New York, 1980; pp 613-615.

(52) Simonneaux, G.; Bondon, A. Mechanism of electron transfer in heme proteins and models: the NMR approach. Chem. Rev. 2005, 105, 2627-2646.

(53) Rajagopal, B. S.; Edzuma, A. N.; Hough, M. A.; Blundell, K. L. I. M.; Kagan, V. E.; Kapralov, A. A.; Fraser, L. A.; Butt, J. N.; Silkstone, G. G.; Wilson, M. T.; Svistunenko, D. A.; Worrall, J. A. R. The hydrogen-peroxide-induced radical behavior in human cytochrome $c$ phospholipid complexes: implications for the enhanced pro-apoptotic activity of the G41S mutant. Biochem. J. 2013, 456, 441-452.

(54) McGovern, R. E.; Feifel, S. C.; Lisdat, F.; Crowley, P. B. Microscale Crystals of Cytochrome $c$ and Calixarene on Electrodes: Interprotein Electron Transfer between Defined Sites. Angew. Chem., Int. Ed. 2015, 54, 6356-6359.

(55) Jeng, W. Y.; Chen, C. Y.; Chang, H. C.; Chuang, W. J. Expression and characterization of recombinant human cytochrome $c$ in E. coli. J. Bioenerg. Biomembr. 2002, 34, 423-431.

(56) Rivera, M.; Walker, F. A. Biosynthetic preparation of isotypically labeled heme. Anal. Biochem. 1995, 230, 295-302.

(57) Arslan, E.; Schulz, H.; Zufferey, R.; Künzler, P.; Thöny-Meyer, L. Overproduction of the Bradyrhizobiumjaponicumc-type cytochrome subunits of the cbb3 oxidase in Escherichia coli. Biochem. Biophys. Res. Commun. 1998, 251, 744-747.

(58) Barker, P. D.; Bertini, I.; Del Conte, R.; Ferguson, S. J.; Hajieva, P.; Tomlinson, E.; Turano, P.; Viezzoli, M. S. A further clue to understanding the mobility of mitochondrial yeast cytochrome c: a (15) N T1rho investigation of the oxidized and reduced species. Eur. J. Biochem. 2001, 268, 4468-4476.

(59) Laviron, E. General expression of the linear potential sweep voltammogram in the case of diffusionless electrochemical systems. J. Electroanal. Chem. Interfacial Electrochem. 1979, 101, 19-28. 